\title{
Health-related quality of life, anxiety and depression in thyroid cancer patients under short-term hypothyroidism and TSH-suppressive levothyroxine treatment
}

Sefik Tagay, Stephan Herpertz ${ }^{2}$, Matthias Langkafel, Yesim Erim, Lutz Freudenberg ${ }^{2}$, Nicole Schöpper ${ }^{1}$, Andreas Bockisch ${ }^{1}$, Wolfgang Senf and Rainer Görges ${ }^{1}$

Clinic of Psychosomatic Medicine and Psychotherapy and ${ }^{1}$ Department of Nuclear Medicine, University of Duisburg-Essen, Essen, Germany and

${ }^{2}$ Department of Psychosomatic Medicine and Psychotherapy, Ruhr-University Bochum, Germany

(Correspondence should be addressed to S Tagay, Clinic of Psychosomatic Medicine and Psychotherapy, University of Duisburg-Essen, Virchowstr. 174, 45147 Essen, Germany; Email: sefik.tagay@uni-essen.de)

\begin{abstract}
Objective: Very few previous studies have compared the degree of health-related quality of life (HRQL), depression and anxiety of differentiated thyroid cancer patients (DTC) under short-term hypothyroidism and levothyroxine treatment.

Methods: Using patient-completed instruments, we examined the frequency of physical complaints, HRQL, anxiety and depression in 130 DTC patients hospitalized for radioiodine therapy or wholebody diagnostics (age 52 years, female 71\%) under short-term hypothyroidism (4 weeks of levothyroxine withdrawal; DTC-H) and in 100 DTC out-patients under TSH-suppressive doses of levothyroxine subsequent to radioiodine therapy (DTC-L; age 49 years, female $81 \%$ ).

Results: Compared with the German general population, DTC-H as well as DTC-L patients had significantly impaired HRQL. Notably, the decrease in HRQL was significantly higher in DTC-H than in DTCL patients. Surprisingly, the prevalence of anxiety $(44.6 \%)$ but not depression $(17.7 \%)$ was much higher in the DTC-H patients than in the general population. In contrast to expectations, similar results for anxiety $(44.0 \%)$ and depression $(17.6 \%)$ were observed in the DTC-L patients.

Conclusions: This mounting evidence suggests that a consistent pattern of HRQL impairment is experienced by patients with DTC. The high frequency of anxiety and the significantly reduced HRQL should be considered in the aftercare of DTC patients.
\end{abstract}

European Journal of Endocrinology 153 755-763

\section{Introduction}

Thyroid cancer accounts for about $1 \%$ of all malignancies and for $0.2 \%$ of cancer deaths. It is the most common malignancy of the endocrine system. Differentiated thyroid cancer (DTC) is composed of papillary or follicular cells and has a generally good prognosis. The standard treatment for DTC is total thyroidectomy, if necessary with cervical lymph node dissection, followed by radioiodine ablation (with the exception of papillary microcarcinoma). Further, standard treatment consists of lifelong supplementation with levothyroxine at a dose titrated to suppress thyrotropin (TSH) levels close to the detection level. The overall long-term worldwide survival rate of patients with thyroid cancer is more than $90 \%$, with variations among subsets of patients (1). However, up to $20 \%$ of patients develop local or locoregional recurrence and up to $13 \%$ develop distant metastases, as long as decades after the initial diagnosis (2). In the long-term management of cancer, assessment of health-related quality of life (HRQL) issues in cancer patients has been exponentially increasing in recent years. The aims of cancer treatment are not only to increase survival but also to preserve HRQL (3). Previous studies of HRQL among thyroid cancer patients which have been confined to the period of withdrawal of thyroid hormone medication had limited sample sizes, and evaluated limited aspects of quality of life (4-6). Yet, few studies have been performed following surgical and radiosotope treatment of thyroid cancer. This may be due to the associated low mortality and morbidity rates. Indeed, health care providers tend to regard DTC somewhat nonchalantly, especially when compared with non-thyroidal cancer of the head and neck. However, resulting side-effects of surgical, radioactive and other means of follow-up testing may nonetheless produce undesirable implications on the patients' general well-being and quality of life. Generic quality of life and associated factors have rarely been investigated in thyroid cancer survivors (7). 
Withdrawal of thyroid hormone therapy with consecutive hypothyroidism has a well-known negative impact on patients' HRQL, leading to increased levels of fatigue, decreased appetite, problems with constipation and motor skills, and fluid retention. Although the efficacy and safety of this approach has been demonstrated for decades, patients frequently complain of symptoms of profound hypothyroidism resulting from levothyroxine withdrawal. Furthermore, during withdrawal, patients show associated psychological symptoms and social changes, such as family distress or decreased motivation to work. Patients suffering from non-metastatic DTC have been shown to have worse HRQL during peak thyroid withdrawal $(5,6)$. Several years ago, recombinant human TSH (rhTSH) was introduced and this avoids the side-effects of thyroid hormone withdrawal (4). The recent availability of rhTSH as a useful method to stimulate radioactive iodine uptake by thyroid remnants $(4,8)$ has stimulated research into the poor psychological performance and loss of HRQL in patients with DTC during levothyroxine withdrawal. Several studies have demonstrated impairment in tests measuring HRQL and psychometric functionality in patients with DTC during tri-iodothyronine (4) or levothyroxine $(5,8)$ withdrawal, compared with these tests when the same patients were taking suppressive doses of thyroid hormones (4), and when these patients were given $\operatorname{rhTSH}(4,8)$.

We therefore performed a cross-sectional study to evaluate physical complaints, HRQL, depression and anxiety in 'cured' DTC out-patients during treatment with chronic suppressive doses of levothyroxine (DTCL) compared with a group of DTC patients under short-term hypothyroidism (DTC-H) hospitalized for radioiodine therapy or whole-body diagnostics. The extent to which HRQL and psychological well-being worsen during levothyroxine withdrawal is still a topic of interest and, more importantly, the possibility that HRQL and mental health may also be impaired by the chronic administration of suppressive doses of levothyroxine has not been satisfactorily explored to date. We hypothesized that DTC-L patients would have significantly better HRQL compared with DTC-H. Further, we expected that DTC-H patients would have significantly more depression and anxiety compared with DTC-L subjects.

\section{Materials and methods}

\section{Study design and patients}

The study consisted of two study samples: a hypothyroid group after 4 weeks of levothyroxine withdrawal (DTC-H) and DTC out-patients subsequent to radioiodine therapy under TSH-suppressive levothyroxine treatment (DTC-L). In the DTC-H group, we asked all thyroid cancer patients hospitalized at our center for diagnostic or therapeutic ${ }^{131} \mathrm{I}$ administration under thyroid hormone withdrawal and hence, hypothyroid conditions, at any time from February to December 2001 to complete instruments rating their physical, HRQL and psychological status. Concerning the DTC-L group, consecutive out-patients from February to December 2001 were invited to participate. These patients completed the same instruments as were employed in the DTC-H study. Eligibility criteria included any patients who had undergone total thyroidectomy for well-differentiated thyroid cancer. For both the DTC-H and DTC-L populations, all patients provided written informed consent and the local ethics committee approved the protocol.

\section{Clinical procedures}

Our thyroidectomized DTC patients normally receive levothyroxine in a dose $(\sim 2.5 \mu \mathrm{g} / \mathrm{kg}$ body weight $)$ that suppresses endogenous TSH levels. In patients undergoing thyroid hormone withdrawal, levothyroxine treatment was discontinued 4 weeks before radioiodine. According to German radioprotection law, patients receiving radioiodine must be hospitalized for at least $48 \mathrm{~h}$. On day 1 of hospitalization, clinical examination, neck ultrasonography and measurement of TSH, free thyroid hormones, Tg and routine clinical chemistry were performed, and radioiodine was given orally as a capsule in the early afternoon. Radioiodine whole-body scan was performed 3-10 days after radioiodine administration. All hormones were measured with commercially available kits (B.R.A.H.M.S. Diagnostika GmbH, Henningsdorf, Germany), TSH with an immunoradiometric coated tube sandwich assay (DYNOtest BRAHMS Diagnostica, Henningsdorf, Germany TSH1) and free thyroxine (fT4) and free tri-iodothyronine (fT3) with competitive solid phase antigen radioimmunometric assays (DYNOtest FT4 and DYNOtest FT3). Reference ranges were 0.3$4.0 \mathrm{mU} / \mathrm{l}$ for TSH, $7.8-19.4 \mathrm{pmol} / \mathrm{l}$ for fT4 and 3.5$8.1 \mathrm{pmol} / \mathrm{l}$ for fT3.

\section{Study instruments}

The health survey short-form 36 (SF-36) The health survey short-form 36 (SF-36) (9) was used to assess generic HRQL. This generic instrument covers aspects of physical, psychological and social functioning (10). It measures four domains in the area of physical health: physical functioning, role-physical, bodily pain and general health and four domains in the area of mental health: role-emotional, vitality, mental health and social functioning. Two comprehensive indices of HRQL were also computed (physical component summary (PCS) and mental component summary (MCS)). The responses to the questions in each domain are added to provide eight scores between 0 and 100 with higher scores reflecting better HRQL. We compared the SF-36 scores of the present study with 
those of a large $(n=2911)$ German general population group (11).

The hospital anxiety and depression scale (HADS) To assess depression and anxiety, the study used the HADS, a 14-item questionnaire that is particularly suitable for somatically ill patients (12). Subjects rate each item on a four-point Likert scale ranging from 0 (not present) to 3 (always present), with higher scores indicating a greater degree of mood disturbance. As recommended by Zigmond \& Snaith (12), we grouped our patients according to their subscale scores as non-cases $(0-7$ score), as borderline cases $(8-10$ score) or as definite cases (11-21 score). Data from a German version (HADS-D) from a general German population as well as from large international medically ill populations have been reported (13).

Beck depression inventory (BDI) Also used to assess depression, specifically, and its cognitive and physical symptoms, was this 21-item questionnaire (14), in which each item is scored on a four-point scale, with higher scores denoting greater severity. Total scores on the instrument of $\leq 10$ reflect normal mood variation and of $\geq 11$ increasing degrees of clinical depression.

Profile of mood states (POMS) To assess overall psychological distress and specific mood states, the study used this 65-item scale (15) which asks patients to describe their mood over the previous week with adjectives rated from 0 (not at all) to 4 (extremely). The instrument contains six subscales originally derived from factor analytic studies: fatigue-inertia, depression-dejection, vigor-activity, confusion-bewilderment, tension-anxiety and anger-hostility. To capture the patient's overall psychological status on the POMS, we used the total mood disturbance (TMD) score, which is the sum of the five negative mood state subscales, minus the vigor subscale. The validity and reliability of the POMS have been documented extensively, especially in cancer (16).

Hypothyroid physical complaints scale Clinical hypothyroidism was evaluated by a self-rating scale comprising classical hypothyroid symptoms, based on the Zulewski score (17) and Billewicz score (18), which have been used in numerous previous studies $(4,8)$.

\section{Statistics}

Data derived from descriptive statistical analyses are presented in the form of percentages or categorial variables and of the mean and S.D. for continuous data. Categorial data were compared using the chi ${ }^{2}$-test and continuous data by the Student's $t$-test and ANOVA or, if appropriate, by non-parametric tests. Significant post hoc comparisons included the Bonferroni modification $(P<0.01)$. The level of significance was set at 0.05; all tests were formulated two-tailed. All statistical analyses were made with the Statistical Package for the Social Sciences (SPSS, Release 11.0, Chicago 2001).

\section{Results \\ Patient characteristics}

The demographic characteristics of the patients in the two samples are shown in Table 1. Of 160 eligible patients, $141(88 \%)$ agreed to participate in the hypothyroid population study, four (3\%) refused and $15(9 \%)$ were excluded because of insufficient German language proficiency to understand the study instruments. Data from five (4\%) of the 141 participants had to be discarded because of very incomplete responses. Six patients who received T3 for 2 weeks were eliminated from the study because their period of hypothyroidism and the degree was shorter. Thus, 130 patients $(81.2 \%$ of eligible patients) were ultimately included in the DTC-H group. All had been thyroidectomized for DTC before study entry. Their median serum TSH concentration on day 1 of hospitalization was $75 \mathrm{mU} / \mathrm{l}$ (range: 17-192 mU/l). At that time, all patients under thyroid hormone withdrawal were biochemically hypothyroid (fT4, fT3 or both below normal range). Of 140 eligible out-patients under TSH-suppressive levothyroxine treatment, $111(79.2 \%)$ agreed to participate in the L-thyroxine population study, six $(4.2 \%)$ were excluded because of insufficient German language. Data from five (3.5\%) of the 111 patients had to be discarded because of very incomplete responses. There was no statistically significant difference between the DTC-H and DTC-L groups in terms of age, gender, marital status, education and employment status. Thus, both groups were comparable.

In both groups, most thyroid cancer patients reported being married, more than half were working and approximately two-thirds were women. Most DTC patients had a diagnosis of papillary thyroid cancer (68.3 vs $80 \%$ respectively). Only in histology did we observe significant group differences $(P<0.047)$. As expected, significant differences were found in TSH $(P<0.001)$, fT3 $(P<0.001)$ and fT4 $(P<0.001)$.

\section{Hypothyroid patients (DTC-H): patients undergoing a diagnostic procedure (whole- body scan) vs patients receiving high-dose radioiodine treatment}

Ninety $(69 \%)$ of the DTC-H patients received ${ }^{131}$ I treatment and $40(31 \%)$ were undergoing a whole-body scan (Table 2). Both groups were similar with respect to the studied outcomes. No significant group differences could be found with respect to depression and anxiety, TMD and HRQL. Furthermore, there were no significant differences in age $(P<0.341)$ and gender $(P<0.168)$. Only in the domain of 
Table 1 Sociodemographic characteristics and biochemical variables.

\begin{tabular}{|c|c|c|c|}
\hline & DTC-H $n=130$ & DTC-L $n=100$ & $\boldsymbol{P}$ \\
\hline Age (years) (S.D.) & $52.3(16.4)$ & $49.6(15.7)$ & 0.085 \\
\hline Gender (\%) & & & 0.207 \\
\hline Women & 71.0 & 81.0 & \\
\hline Martial status (\%) & & & 0.254 \\
\hline Single & 10.0 & 17.2 & \\
\hline Married & 74.6 & 65.7 & \\
\hline Separated/divorced & 3.8 & 7.1 & \\
\hline Widowed & 11.5 & 10.1 & \\
\hline Eduation (\%) & & & 0.635 \\
\hline Other & 2.3 & 3.0 & \\
\hline Secondary General School Certificate & 40.6 & 42.9 & \\
\hline Intermediate School Certificate & 33.6 & 36.7 & \\
\hline University Entrance Diploma & 7.0 & 5.1 & \\
\hline University & 11.7 & 11.2 & \\
\hline Employment status (\%) & & & 0.430 \\
\hline Paid work (full or part time) & 52.4 & 54.0 & \\
\hline Unemployed & 4.8 & 3.4 & \\
\hline Retired & 41.9 & 41.3 & \\
\hline Histology (\%) & & & 0.047 \\
\hline Papillary carcinoma & 68.3 & 80.0 & \\
\hline Follicular carcinoma & 30.7 & 20.0 & \\
\hline \multicolumn{4}{|l|}{ Biochemical variables } \\
\hline TSH level & Median 75 (range 17-192 mU/l) & Median $<0.01$ (range $<0.1-0.3 \mathrm{mU} / \mathrm{l})$ & 0.001 \\
\hline $\mathrm{fT} 4$ & Median $3.5($ range $<2.5-11 \mathrm{pmol} / \mathrm{l})$ & Median 20.5 (range $13.2-28.9 \mathrm{pmol} / \mathrm{l})$ & 0.001 \\
\hline fT3 & Median $<2.8($ range $<2.8-4.3 \mathrm{pmol} / \mathrm{l})$ & Median 6.3 (range $3.8-11.1 \mathrm{pmol} / \mathrm{l})$ & 0.001 \\
\hline
\end{tabular}

the SF-36 'bodily pain' group were differences found at the $5 \%$ significance level. Overall, it is justified to summarize both groups as one subsample.

\section{Hypothyroid symptoms: hypothyroid group (DTC-H) and L-thyroxine group (DTC-L)}

As expected, hypoythyroid patients were significantly more likely than L-thyroxine patients to express symptoms of thyroid dysregulation in nine of twelve areas of symptoms (Table 3). The DTC-H group had higher prevalences in slow movements $(P<0.05)$, weight increase $(P<0.000)$, cold skin $(P<0.000)$, parasthesia $(P<0.000)$, freeze $(P<0.001)$, cold intolerance $(P<0.026)$, periorbital puffiness $(P<0.000)$, hoarseness $(P<0.024)$ and constipation $(P<0.023)$ than the DTC-L group. There were no statistical differences between both groups in the prevalence of coarse

Table 2 DTC-H patients: whole body diagnostic vs therapy patients receiving active treatment (radioiodine).

\begin{tabular}{|c|c|c|c|c|}
\hline & Diagnostic patients Mean (S.D.) & Therapy patients Mean (S.D.) & $t$-test & $P$ \\
\hline \multicolumn{5}{|l|}{ Hospital anxiety and depression scale } \\
\hline HADS depression & $4.2(3.4)$ & $4.4(3.7)$ & 0.25 & 0.804 \\
\hline HADS anxiety & $7.6(3.7)$ & $7.2(3.6)$ & 0.56 & 0.579 \\
\hline \multicolumn{5}{|l|}{ Beck depression inventory } \\
\hline \multirow{2}{*}{\multicolumn{5}{|c|}{ Profile of mood states }} \\
\hline & & & & \\
\hline POMS depression-dejection & $1.4(0.34)$ & $1.5(0.37)$ & 0.67 & 0.504 \\
\hline POMS tension-anxiety & $1.2(0.74)$ & $1.2(0.82)$ & 0.05 & 0.961 \\
\hline POMS anger-hostility & $1.2(0.44)$ & $1.2(0.44)$ & 0.08 & 0.930 \\
\hline POMS vigor-vitality & $1.3(0.49)$ & $1.3(0.43)$ & 0.79 & 0.427 \\
\hline POMS fatigue-intertia & $1.1(0.51)$ & $1.1(0.56)$ & 0.49 & 0.621 \\
\hline POMS confusion-bewilderment & $0.76(0.69)$ & $0.73(0.63)$ & 0.19 & 0.845 \\
\hline \multicolumn{5}{|l|}{ Health survey short form SF-36 } \\
\hline Physical function & $63.8(27.3)$ & $63.8(29.6)$ & 0.01 & 0.991 \\
\hline Role-physical & $48.6(40.3)$ & $38.5(39.6)$ & 1.2 & 0.217 \\
\hline Bodily pain & $64.5(31.9)$ & $75.6(27.4)$ & 2.0 & 0.044 \\
\hline General health & $58.3(21.1)$ & $59.7(20.3)$ & 0.34 & 0.732 \\
\hline Vitality & $40.2(22.6)$ & $41.9(23.3)$ & 0.40 & 0.689 \\
\hline Social functioning & $62.8(22.6)$ & $59.3(26.4)$ & 0.69 & 0.490 \\
\hline Role-emotional & $45.0(45.3)$ & $52.6(45.1)$ & 0.84 & 0.399 \\
\hline Mental health & $58.3(20.9)$ & $62.3(20.8)$ & 0.97 & 0.334 \\
\hline
\end{tabular}

www.eje-online.org 
Table 3 Prevalence of hypothyroid symptoms and signs.

\begin{tabular}{lccrc}
\hline Physical complaint & DTC-H (\%) & DTC-L $(\%)$ & Chi $^{2}$ & $\boldsymbol{P}$ \\
\hline Slow movements & 61 & 48 & 3.8 & 0.050 \\
Weight increase & 61 & 31 & 19.2 & 0.000 \\
Cold skin & 61 & 48 & 16.5 & 0.000 \\
Parathesia & 56 & 25 & 19.8 & 0.000 \\
Freeze & 56 & 38 & 6.4 & 0.001 \\
Cold intolerance & 48 & 32 & 4.9 & 0.026 \\
Periorbital puffiness & 48 & 21 & 16.5 & 0.000 \\
Hoarseness & 36 & 22 & 5.1 & 0.024 \\
Coarse skin & 27 & 16 & 3.4 & 0.062 \\
Hearing impairment & 24 & 18 & 1.2 & 0.260 \\
Constipation & 22 & 10 & 5.1 & 0.023 \\
\hline
\end{tabular}

skin and hearing impairment. Most frequent symptoms in the DTC-H patients were slow movements, weight increase and cold skin (all 61\%).

\section{Psychological distress: depression, anxiety and mood disturbance}

No significant difference could be observed between the DTC-H group and the DTC-L group with regard to depression according to HADS-D $(4.3 \pm 3.6$ vs $4.1 \pm 3.8 ; P<0.682)$ and BDI $(8.4 \pm 7.0$ vs $7.2 \pm 6.9$; $P<0.289$ ). Interestingly, for both groups, mean \pm S.D. depression scores were in the normal or non-clinically relevant range on the HADS and BDI as well as in POMS depression subscale. Again, differences between both groups failed statistical significance in most cases (Table 4). Only in the POMS was the subscale 'tension-anxiety' increased significantly in the DTC-H group ( $1.1 \pm 0.80$ vs $0.88 \pm 0.66 ; P<0.012)$. In contrast, the subscale 'vigor-vitality' was decreased in DTC-H patients $(1.3 \pm 0.41$ vs $1.1 \pm 0.45 ; P<0.000)$. Overall, there were no group differences between DTC-H patients and DTC-L group population in depression, anxiety and in most areas of current mood disturbances.
Table 5 presents the cases of depression and anxiety in the both groups. According to the cut-off criteria of the HADS-D, 9.2\% of the DTC-H patients were identified as borderline depression cases and another $8.5 \%$ as definite depression cases, for a probable prevalence of clinical depression of $17.7 \%$. In contrast, for anxiety $23.1 \%$ of DTC-H patients were identified as borderline anxiety cases and $21.5 \%$ as definite anxiety cases on the HADS anxiety scale, for an overall probable prevalence of anxiety of $44.6 \%$. The DTC-L group patients had similar prevalences as the DTC-H group in the cases of depression and anxiety. Again, there were no statistical differences between both groups with regard to the depression and anxiety cases.

\section{HRQL}

Comparison of HRQL in DTC patients with a German reference population Mean values for all domains of the SF-36 scales for the three groups are depicted in Table 6 . The representative German reference group consisted of 2914 subjects. The mean age of all participants was 47.3 years, ranging in age from 14 to 74 years; $55.6 \%$ were women and $77.6 \%$ of the subjects were married or cohabiting. Approximately 30\% had a Secondary General School Certificate. Almost 30\% were in a full-time job, nearly 30\% were retired (11). Both study samples ware compared with the reference population matching for age. In seven of eight domains of the SF-36, DTC-H patients reported significantly reduced HRQL compared with the German reference group adjusted for age (each variable $P<0.001$, expect bodily pain with $P<0.01)$. There was no significant difference between DTC-H patients and the reference group with regard to general health. Overall, mental health, as reflected by the SF-36 MCS, was significantly lower in the hypothyroid DTC patients compared with the reference group. Furthermore, compared with the German reference population, the DTC-L patients had significantly lower scores in seven of eight SF-36 domains and in the

Table 4 Depression, anxiety and profile of mood states of the study populations.

\begin{tabular}{|c|c|c|c|c|}
\hline & DTC-H Mean (S.D.) & DTC-L Mean (S.D.) & $t$-test & $P$ \\
\hline \multicolumn{5}{|l|}{ Hospital anxiety and depression scale } \\
\hline HADS depression & $4.3(3.6)$ & $4.1(3.8)$ & 0.410 & 0.682 \\
\hline HADS anxiety & $7.2(3.6)$ & $7.2(3.4)$ & 0.008 & 0.993 \\
\hline \multicolumn{5}{|l|}{ Beck depression inventory } \\
\hline BDI-depression & $8.4(7.0)$ & $7.2(6.9)$ & 1.1 & 0.289 \\
\hline \multicolumn{5}{|l|}{ Profile of mood states } \\
\hline POMS depression-dejection & $1.4(0.36)$ & $1.4(0.32)$ & 0.459 & 0.647 \\
\hline POMS tension-anxiety & $1.1(0.80)$ & $0.88(0.66)$ & 2.5 & 0.012 \\
\hline POMS anger-hostility & $1.2(0.44)$ & $1.4(0.32)$ & 0.926 & 0.356 \\
\hline POMS vigor-vitality & $1.1(0.45)$ & $1.3(0.41)$ & 3.6 & 0.000 \\
\hline POMS fatigue-inertia & $1.1(0.54)$ & $1.1(0.54)$ & 0.497 & 0.619 \\
\hline POMS confusion-bewilderment & $0.74(0.64)$ & $0.66(0.67)$ & 0.819 & 0.414 \\
\hline POMS total mood disturbance & $4.1(1.6)$ & $4.1(1.9)$ & 0.163 & 0.871 \\
\hline
\end{tabular}


Table 5 Cases of depression and anxiety (HADS).

\begin{tabular}{lcccc}
\hline & DTC-H (\%) & DTC-L (\%) & Chi $^{2}$ & $\boldsymbol{P}$ \\
\hline HADS depression & & & 1.3 & 0.502 \\
$\quad$ Non case & 82.3 & 82.5 & & \\
$\quad$ Borderline case & 9.2 & 12.4 & & \\
$\quad$ Definite case & 8.5 & 5.2 & \multirow{2}{*}{0.56} & 0.755 \\
HADS anxiety & & & & \\
$\quad$ Non case & 55.4 & 53.0 & & \\
$\quad$ Borderline case & 23.1 & 26.0 & & \\
$\quad$ Definite case & 21.5 & 18.0 & & \\
\hline
\end{tabular}

MCS of SF-36. The difference between the German reference population and the DTC-L group failed statistical significance with regard to bodily pain and PCS.

Comparison of HRQL in DTC-H and DTC-L The differences in favor of the DTC-L group versus the DTC-H group reached significance in physical functioning $(P<0.001)$, role-physical $(P<0.001)$, bodily pain $(P<0.01)$, vitality $(P<0.001)$ and role-emotional $(P<0.001)$. In summary, the PCS $(P<0.001)$ and MCS $(P<0.05)$ of the DCT-L group were better than in the DTC-H group. There was no significant difference between patients with DTC-H and DTC-L with regard to general health, social functioning and mental health. Adjusting the HRQL scores for age, gender and HADS scores confirmed the above-described differences between DTC-L and DTC-H patients.

\section{Discussion}

This is the first study assessing quantitatively and in depth via numerous standardized self-rating instruments, physical complaints, HRQL, depression and anxiety, in a large series of DTC patients undergoing short-term hypothyroidism, and DTC survivals under TSH-suppressive L-thyroxine treatment. The study groups were representative of thyroid cancer patients with regard to distribution of age, gender and histological subtypes (19). DTC is a disease with a generally excellent prognosis. Treatment of DTC is well tolerated, especially compared with other cancer therapeutics, and most patients are advised to expect a completely normal life. Indeed, Dow et al. (5) have suggested that thyroid cancer survivors are considered 'cured' and are a largely forgotten segment of the cancer survivor population. Nevertheless, curative therapy for DTC often involves multimodal interventions and lifelong dependence on exogenous thyroid hormone (2).

We found that the short-term hypothyroidism resulting from thyroid hormone withdrawal causes physical symptoms in the majority of patients. Compared with DTC-L patients, DTC-H patients had a significantly higher prevalence of virtually all hypothyroid symptoms listed on the test instrument. These observations are in line with those of quantitative $(4,8,20,21)$ and qualitative $(7,22)$ studies in the same setting. It is generally known that patients with thyroid cancer suffer troublesome physical symptoms relating to short-term hormone withdrawal $(5,7)$. The less-apparent negative psychological, family and work sequelae have also been demonstrated $(5,6)$. These sequelae include physical symptoms, impaired psychological well-being, family distress, decreased motivation to work and decreased quality of work.

Our assessment of depression and anxiety in the present study revealed two surprising findings. First, as measured by the mean HADS depression subscale score, the severity of depressive symptoms in our large DTC-H patient population and in the DTC-L survivals was in the normal range for healthy reference populations (12). Additionally, depression case prevalence as defined by HADS criteria was comparable between our thyroid cancer patients and the reference population (12). Moreover, there was no significant difference in the severity or case prevalence of depression between the DTC-H and the DTC-L groups.

The lack of an abnormal degree of depression in both study groups, hypothyroid and DTC survival populations, was confirmed by the mean POMS depression

Table 6 Mean and S.D. of SF-36 HRQL scores: German reference population* and DTC-L and DTC-H groups.

\begin{tabular}{|c|c|c|c|c|c|c|c|}
\hline \multirow[b]{2}{*}{ Physical function } & \multicolumn{2}{|c|}{$\begin{array}{l}\text { German reference population } \\
\text { (age: } 41-50 \text { years) }\end{array}$} & \multirow{2}{*}{$\frac{\text { DTC-L }}{78.4 \pm 24.3}$} & \multicolumn{3}{|c|}{ DTC-H } & \multirow{2}{*}{$\begin{array}{c}\text { German reference population } \\
\text { (age: } 51-60 \text { years)* }^{*} \\
83.7 \pm 19.5\end{array}$} \\
\hline & $88.9 \pm 17.6$ & $\gg$ & & $\gg$ & $63.8 \pm 28.8$ & 《 & \\
\hline Role-physical & $87.4 \pm 27.6$ & $\gg$ & $74.4 \pm 36.2$ & $\gg$ & $41.8 \pm 39.9$ & 《 & $80.5 \pm 31.8$ \\
\hline Bodily pain & $72.7 \pm 27.3$ & $=$ & $82.4 \pm 26.8$ & $\geq$ & $72.2 \pm 29.2$ & $\leq$ & $78.9 \pm 28.0$ \\
\hline General health & $68.0 \pm 18.8$ & $\geq$ & $61.3 \pm 22.3$ & $=$ & $59.3 \pm 20.5$ & $=$ & $61.0 \pm 19.2$ \\
\hline PCS & $50.9 \pm 9.2$ & $=$ & $49.9 \pm 9.6$ & $\gg$ & $44.2 \pm 9.7$ & $=$ & $47.8 \pm 17.3$ \\
\hline Social functioning & $89.3 \pm 17.3$ & $\gg$ & $64.2 \pm 24.2$ & $=$ & $60.3 \pm 25.3$ & $\ll$ & $86.8 \pm 18.0$ \\
\hline Role-emotional & $91.5 \pm 22.7$ & $》$ & $77.5 \pm 36.6$ & $\gg$ & $50.2 \pm 45.1$ & $\ll$ & $88.8 \pm 25.7$ \\
\hline Mental health & $73.6 \pm 15.5$ & $\gg$ & $65.0 \pm 18.2$ & $=$ & $61.1 \pm 20.8$ & $\ll$ & $72.4 \pm 16.1$ \\
\hline MCS & $51.5 \pm 7.4$ & $》$ & $44.3 \pm 9.7$ & $>$ & $40.8 \pm 10.4$ & 《 & $51.1 \pm 8.1$ \\
\hline
\end{tabular}

$=$, not significant; $>, P<0.05, \leq$ and $\geq P<0.01 ; 《$ and $》, P<0.001$.

* Reported in reference 11. 
and BDI scores, both fell into the "not clinically relevant' range. In contrast to our findings, other studies have found significantly (20) and numerically (4) higher scores on the POMS depression subscale in DTC patients when they undergo short-term hypothyroidism than when they are euthyroid. The association between long-term thyroid hormone deficiency and clinical depression, even when the hypothyroidism is subclinical, has long been known and is well documented (23): clinical depression occurs in $>40 \%$ of chronically hypothyroid patients. Hypothyroidism, even in a mild form, is clearly associated with neurocognitive dysfunction including memory loss and depression (24). Taken together with the results of earlier DTC studies $(4,20)$, our observations suggest that the association between short-term hypothyroidism and depression is less clear. Multiple factors may be involved for the different prevalence of depression in short-term hypothyroidism, including differences in diagnostic criteria, assessment procedures and the sample characteristics. The heterogeneity of depression is probably an important factor as well. Additional possible factors are unclear sources of the reference values used and small sample sizes.

The second unexpected finding of the psychological testing in our study is the high prevalence of anxiety symptoms in DTC patients. We found that both the DTC-H and DTC-L patients had higher anxiety scores than the German reference population, as assessed by the HADS anxiety score.

While, in general, anxiety is even more frequent in oncological patients than is depression (25), the high rate of HADS anxiety cases in our study groups compared with both selected and unselected samples of cancer patients (26) is remarkable. We found a $23 \%$ prevalence of borderline cases in our DTC-H population while, for example, Moorey et al. (27) reported a $27 \%$ and Skarstein et al. (26) a 13\% prevalence within patients with non-thyroid cancers, although most of these cancer types are associated with poorer prognoses and more invasive interventions. We noted that another $21 \%$ of our DTC-H population were definite HADS anxiety cases, while the analogous prevalences ranged from $6 \%$ in successfully treated patients with testicular cancer (28) to $19 \%$ in patients with various types of non-thyroid cancers in a radiotherapy unit (25). Moreover, DTC-L patients in our study showed similar frequencies in borderline cases $(26 \%)$ as well as in definite cases (18\%).

In contrast to our hypothesis, significant differences in depression and anxiety between DTC-H and DTC-L groups were not observed. The data in the literature concerning this topic are inconsistent and only published for chronic hypothyroidism in non-DTC patients, which is not necessarily comparable with short-term hypothyroidism after 4 weeks of levothyroxine withdrawal in DTC patients. In a consecutive sample, Monzani et al. (29) found increased anxiety in subclinical hypothyroid patients. The authors claimed that the cause of higher anxiety scores in subclinical hypothyroid subjects might be minor neurobehavioral disturbances. Another study related to subclinical hypothyroidism and anxiety was reported by Baldini et al. (30) who evaluated the effect of subclinical hypothyroidism on psychopathological functions including anxiety in female patients and euthyroid female goiter controls. No significant difference in anxious symptomatology was detected between the groups. More recently, Engum et al. (31) performed a field research study in Norway. They reported that there is no significantly increased risk of anxiety in subclinical hypothyroidism and hyperthyroidism. The results of the study by Engum et al. (31) is consistent with that by Baldini et al. (30). Demet et al. (32) determined the prevalence and severity of depression and anxiety in patients with hypothyroidism and compared these with euthyroid patients. Thirty patients with hypothyroidism and 30 euthyroid controls were recruited. There was no difference between the two groups. Therefore, depression and anxiety were not outstanding features in hypothyroidism. However, depression was more significant in the chronically hypothyroid than in the euthyroid group. In conclusion, these findings demonstrated that the relations between thyroid functional state and anxiety are less clear.

Environmental factors such as uncertainty about the diagnostic results (i.e. detection of relapses) and especially for the DTC-H group - hospitalization under radiation protection and surrounding radioiodine diagnostic and treatment procedures may help to explain the high rate of anxiety in our study populations. As Dow et al. (5) and Stajduhar et al. (22) point out, patients awaiting the results of the diagnostic procedures or kept in isolated conditions are usually extremely stressed; anxiety could be a correlative of this stress. A causal relationship between short-term hypothyroidism and anxiety seems unlikely, since the mean degree and the case frequency of anxiety were similar in the DTC-L study. Future investigation is necessary to analyze the cause of anxiety in the DTC patients. A clinical implication of our findings is that depression and anxiety appear and therefore should be treated independently from the thyroid functional state. Moreover, given its surprisingly high prevalence in both DTC-H and DTC-L patients, anxiety should be strongly considered in the aftercare of DTC patients. We found that HRQL is considerably reduced both in physical and psychosocial dimensions in thyroid cancer patients undergoing short-term hypothyroidism as well as in DTC-L patients, compared with a healthy reference population. These HRQL impairments appear to be largely due to hypothyroidism, since our DTC survivals had reduced HRQL scores compared with those of the reference population. Moreover, the DTC-L patients had significantly better HRQL in most domains of the SF-36 than did their DTC-H counterparts. 
Compared with the general population, DTC-H group scored lower in all domains of the SF-36. Thus, based upon the now available data, there is irrefutable evidence for a significant impairment of HRQL in DTC patients. This impairment is at least as severe as the impairment in other chronic diseases.

In the past, various studies have reported a link between reduced HRQL and DTC (8, 21, 33, 34). HRQL is now considered an important endpoint in clincial studies (35). Our results dovetail with those of two studies examining HRQL in the thyroid cancer population. Recently, a Spanish study showed that, when hypothyroid, DTC patients had lower HRQL scores than healthy controls. However, when euthyroid, the same individuals had similar HRQL scores to those of the healthy controls, except on the general health functional scale, which was lower in the DTC patients (21). In an Austrian study (33) of 150 consecutive DTC patients on levothyroxine, SF-36 HRQL functional scores were statistically similar between the DTC patients and sex- and age-matched healthy controls in all SF-36 functional areas except role-emotional and vitality. Of interest, in the Austrian study, the SF-36 role-physical, mental health and social functioning scores were also significantly lower in the subgroup that had been diagnosed with DTC within 1 year of study entry than in controls. This suggested an HRQL improvement in DTC patients with time since diagnosis independent from their metabolic state. Hence, the length of this interval in a given study population could influence the study's HRQL findings. Most studies concerning HRQL issues during withdrawal of thyroid hormone therapy have not used generic or specific HRQL tools (Billewicz scale, POMS) but have included quite small samples $(5,6)$. These studies have focused especially on impairments of HRQL during withdrawal of thyroid hormone therapy $(5,6)$. Dow et al. (5) studied HRQL after withdrawal of thyroid hormone therapy, utilizing an instrument designed specifically for this purpose which was tested and validated. The majority of HRQL issues addressed in this instrument were general, applicable to most types and locations of cancer. Meier et al. (4) also noted that the most debilitating period in thyroid cancer surveillance was during the induced hypothyroidism for follow-up scanning. The HRQL as measured by two psychometric scales is far better during rhTSH treatment than after thyroid hormone withdrawal. These findings correspond with the results of the present study which indicate a high distress level for thyroid hormone withdrawal. The previous studies of HRQL in DTC patients cited above $(4,8)$ showed a consistent worsening of quality of life during withdrawal of thyroid hormone. Meier et al. (4) used the POMS and found that $94 \%$ of patients with DTC experienced increased fatigue during thyroid hormone withdrawal, and $89 \%$ experienced decreased vigor and activity. Haugen et al. (8) employed the SF-36 health survey and found that many of the parameters evaluated also worsened after levothyroxine withdrawal when compared with treatment with rhTSH. Dow et al. (5) used a specific test for measuring HRQL in patients with DTC during levothyroxine withdrawal (the QoL-thyroid scale), as well as a test for the evaluation of functionality in cancer patients, in a series of 34 patients with DTC. Our present study confirms and expands these results, with the additional advantage of having compared the results of the patients with a control group of DTC patients under L-thyroxine treatment, allowing us to determine the magnitude of the marked deterioration of these variables when compared with DTC controls.

The limitations of the present study should be noted. First, we did not assess the disease-specific HRQL of thyroid cancer. The SF-36 health survey seems to be a very useful instrument to decribe the overall HRQL of patients but, to detect more specific symptoms and HRQL deficits related to different cancer entities, disease-specific questionnaires may be more appropriate (5). Secondly, the psychometric analyses were based on self-rating scales and not on interview data, which may make a difference. Finally, the data are cross-sectional and do not allow us to suggest any causal relationship. Despite these limitations, our results can provide guidance for clinicians dealing with hypothyroid patients, and investigators interested in HRQL impairment in these patients. Patients were drawn consecutively from a large Nuclear Medicine Clinic at a tertiary referral center and should be representative of the DTC general population. All in all, our results have demonstrated a marked deterioration in HRQL and anxiety in patients with DTC during levothyroxine withdrawal as well as in levothyroxine treatment. If confirmed by future studies with DTC patients, the worsening of HRQL and the increased anxiety observed during TSH-suppressive therapy with levothyroxine as well as under short-term hypothyroidism should stimulate research into novel strategies for long-term treatment of patients with DTC.

In summary, our study showed that HRQL was distinctly reduced in DTC patients undergoing thyroid hormone withdrawal to prepare for follow-up diagnostic or therapeutic procedures but - in a minor intensity also in DTC patients under TSH-suppressive levothyroxine treatment. Overall, patients with DTC under shortterm hypothyroidism had more severely impaired HRQL compared with patients with DTC under levothyroxine treatment. Anxiety is considerably more frequent in DTC patients than in the general population and other cancer patient samples, regardless of whether patients had levothyroxine withdrawal or were under levothyroxine treatment. Since short-term hypothyroidism accounts for a substantial proportion of the HQRL impairment, this situation could be improved by avoiding short-term hypothyroidism and applying rhTSH instead. Furthermore, the results of the present study have demonstrated that the increased prevalence of 
anxiety should be considered more in the aftercare of DTC patients, e.g. psycho-oncological support for selected patients. Physicians could help to improve HRQL and reduce the increased anxiety among patients with thyroid cancer by teaching them how to manage these uncomfortable symptoms, informing patients of possible reactions to their cancer, developing a training group for health professionals and designing psychosocial intervention in oncology.

\section{References}

1 Mazzaferri EL. An overview of the management of papillary and follicular thyroid carcinoma. Thyroid 19999 421-427.

2 Schlumberger M. Diagnostic follow up of well-differentiated thyroid carcinoma: historical perspective and current status. Journal of Endocrinological Investigation 199922 3-7.

3 Dow KH \& Lafferty P. Quality of life, survivorship, and psychosocial adjustment of young women with breast cancer after breastconserving surgery and radiation therapy. Oncology Nursing Forum 200027 1555-1564.

4 Meier CA, Braverman LE, Ebner SA, Veronikas I, Daniels GH, Ross DS, Deraska DJ, Davies TF, Valentine M, Degroot LJ, Curan P, McEllin K, Reynolds J, Robbins J \& Weintraub BD. Diagnostic use of recombinant human thyrotropin in patients with thyroid carcinoma (Phase I/II Study). Journal of Clinical Endocrinology and Metabolism $1994 \mathbf{7 8}$ 188-196.

5 Dow KH, Ferrell BR \& Anello C. Quality-of-life changes in patients with thyroid cancer after withdrawal of thyroid hormone therapy. Thyroid 19777 613-619.

6 Dow K, Ferrell B \& Anello C. Balancing demands of cancer surveillance among survivors of thyroid cancer. Cancer Practice $19975289-294$.

7 Dagan T, Bedrin L, Horowitz Z, Chaushu G, Wolf M, Kronenenberg J \& Talmi Y. Quality of life of well-differentiated thyroid carcinoma patients. Journal of Laryngology and Otology $2004118537-542$.

8 Haugen BR, Pacini F, Reiners C, Schlumberger M, Ladenson PW, Sherman SI, Cooper DS, Graham KE, Braverman LE, Skarulis MC, Davies TF, DeGroot LJ, Mazzaferri EL, Daniels GH, Ross DS, Luster M, Samuels MH, Becker DV, Maxon HR 3rd, Cavalieri RR, Spencer CA, McEllin K, Weintraub BD \& Ridgway EC. A comparison of recombinant human thyrotropin and thyroid hormone withdrawal for the detection of thyroid remnant or cancer. Journal of Clinical Endocrinology and Metabolism 199984 3877-3885.

9 Ware JE, Snow KK, Korinski M, Gandeck B. SF-36 Health Survey Manual and Interpretation Guide. Boston: New England Medical Center, The Health Institute, 1993.

10 Bullinger M, Kirschberger I \& Ware JE. The German SF-36 Health Survey. Zeitschrift Gesundheitswissenschaft 199510 21-36.

11 Bullinger M, Kirschberger I. SF-36 Fragebogen zum Gesundheitszustand. Göttingen: Hogrefe, 1998.

12 Zigmond AS \& Snaith RP. The hospital anxiety and depression scale. Acta Psychiatrica Scandinavica 198367 361-370.

13 Herrmann C. International experiences with the hospital anxiety and depression scale - a review of validation data and clinical results. Journal of Psychosomatic Research 199742 17-41.

14 Beck AT, Ward CH, Mendelson M, Mock JE \& Erbaugh JK. An inventory for measuring depression. Archives of General Psychiatry $19614561-571$.

15 McNair DM, Lorr M, Droppeleman LF. EITS Manual for the Profile of Mood States. San Diego CA: Educational and Industrial Testing Service, 1971.

16 Gotoy CC \& Stern JD. Assessment of psychological functioning in cancer-patients. Journal of Psychosocial Oncology 199513 $123-160$.

17 Zulewski H, Müller B, Exer P, Miserez AR \& Staub JJ. Estimation of tissue hypothyroidism by a new clinical score: evaluation of patients with various grades of hypothyroidism and controls. Journal of Clinical Endocrinology and Metabolism 199782 771-776.

18 Billewicz WZ, Chapman RS, Crooks J, Day ME, Gossage J, Wayne E \& Young J. Statistical methods applied to the diagnosis of hypothyroidism. Quarterly Journal of Medicine 1969150 255-266.

19 Görges R. The changing epidemiology of thyroid cancer. In Thyroid Cancer pp 3-27. Eds HJ Biersack \& F Grünwald. Heidelberg: Springer, 2005.

20 Ladenson PW, Braverman LE, Mazzaferri EL, Brucker-Davis F, Cooper DS, Garber JR, Wondisford FE, Davies TF, DeGroot LJ, Daniels GH, Ross DS \& Weintraub BD. Comparison of administration of recombinant human thyrotropin with withdrawal of thyroid hormone for radioactive iodine scanning in patients with thyroid carcinoma. New England Journal of Medicine 1997337 888-896.

21 Botella-Carretero JI, Galán JM, Caballero C, Sanco J \& EscobarMorreale HF. Quality of life and psychometric functionality in patients with differentiated thyroid carcinoma. Endocrine-Related Cancer 200410 601-610.

22 Stajduhar KI, Neithercut J, Chu E, Pham P, Rohde J, Sicotte A \& Young K. Thyroid cancer: patients' experiences of receiving iodine131 therapy. Oncology Nursing Forum 200027 1213-1218.

23 Haggerty JJ, Stern RA, Mason GA, Beckwith J, Morey CE \& Prange AJ. Subclinical hypothyroidism: a modifiable risk factor for depression? American Journal of Psychiatry $1993150508-510$.

24 Dugbartey AT. Neurocognitive aspects of hypothyroidism. Archives of Internal Medicine $1998 \mathbf{1 5 8} 1413-1418$.

25 Maher EJ, Mackenzie C, Young T \& Marks D. The use of the hospital anxiety and depression scale (HADS) and the EORTC QLQC30 questionnaire to screen for treatable unmet needs in patients attending routinely for radiotherapy. Cancer Treatment Reviews 199622 123-129.

26 Skarstein J, Aass N, Fossa S, Skovlund E \& Dahl A. Anxiety and depression in cancer patients: relation between the hospital anxiety and depression scale and the European organization for research and treatment of cancer core quality of life questionnaire. Journal of Psychosomatic Research 200049 27-34.

27 Moorey S, Greer S, Watson M, Gorman C, Rowden L, Tunmore R, Robertson B \& Bliss J. The factor structure and factor stability of the hospital anxiety and depression scale in patients with cancer. British Journal of Psychiatry 1991158 255-259.

28 Fossa SD \& Dahl AA. Short form 36 and hospital anxiety and depression scale - a comparison based on patients with testicular cancer. Journal of Psychosomatic Research 200252 79-87.

29 Monzani F, Del Guerra P, Caraccio N, Pruneti CA, Pucci E, Luisi M \& Baschieri L. Subclinical hypothyroidism: neurobehavioral features and beneficial effect of l-thyroxine treatment. Clinical Investigation 199371 367-371.

30 Baldini IM, Vita A, Mauri CM, Amodei V, Carrisi M, Bravin S \& Cantalamessa L. Psychopathological and cognitive features in subclinical hypotyhroidism. Progress in Neuro-Psychopharmacology and Biological Psychiatry 199721 925-935.

31 Engum M, Bjoro T, Mykletun A \& Dahl AA. An association between depression, anxiety and thyroid function - a clinical fact or artifact? Acta Psychiatrica Scandinavica 2002106 27-34.

32 Demet NM, Ozmen B, Deveci A, Boyvada S, Adiguzel H \& Aydemir O. Depression and anxiety in hypotyhroidism. West Indian Medical Journal 200352 223-227.

33 Crevenna R, Zettinig G, Keilani M, Posch M, Schmidinger M, Pirich C, Nuhr M, Wolzt M, Quittan M, Fialka-Moser V \& Dudczak R. Quality of life in patients with non-metastatic differentiated thyroid cancer under thyroxine supplementation therapy. Support Care Cancer 200411 597-603.

34 Schultz PN, Stava C \& Vassilopoulou-Sellin R. Health profiles and quality of life of 518 survivors of thyroid cancer. Head and Neck $200325349-356$.

35 Testa MA. A pilot study of life-outcomes. European Journal of Medicine $20009230-234$.

Received 8 February 2005

Accepted 13 September 2005 